\title{
Speeding up the Dreyfus-Wagner Algorithm for minimum Steiner trees
}

\author{
Bernhard Fuchs* \\ Center for Applied Computer Science Cologne (ZAIK) \\ University of Cologne, Weyertal 80, 50931 Köln, Germany \\ Walter Kern ${ }^{\dagger}$ Xinhui Wang $\ddagger$ \\ University of Twente, Department of Applied Mathematics \\ Faculty of EEMCS, P.O.Box 217 \\ 7500 AE Enschede, The Netherlands
}

\begin{abstract}
The Dreyfus-Wagner algorithm is a well-known dynamic programming method for computing minimum Steiner trees in general weighted graphs in time $O^{*}\left(3^{k}\right)$, where $k$ is the number of terminal nodes to be connected. We improve its running time to $O^{*}\left(2.684^{k}\right)$ by showing that the optimum Steiner tree $T$ can be partitioned into $T=T_{1} \cup T_{2} \cup T_{3}$ in a certain way such that each $T_{i}$ is a minimum Steiner tree in a suitable contracted graph $G_{i}$ with less than $\frac{k}{2}$ terminals. In the rectilinear case, there exists a variant of the dynamic programming method that runs in $O^{*}\left(2.386^{k}\right)$. In this case, our splitting technique yields an improvement to $O^{*}\left(2.335^{k}\right)$.
\end{abstract}

\section{Introduction}

The Steiner tree problem is one of the most well-known NP-hard problems: Given a graph $G=(V, E)$ of order $n=|V|$, edge $\operatorname{costs} c \in \mathbb{R}_{+}^{E}$ and a set

*Email: bfuchs@zpr.uni-koeln.de

${ }^{\dagger}$ Email: kern@math.utwente.nl

${ }_{\ddagger}^{\ddagger}$ Email: xinhuiw@math.utwente.nl 
$S \subseteq V$ of $k=|S|$ terminal nodes, we are to find a minimum cost subtree $T=T(S)$ of $G$ connecting (spanning) all terminal nodes. Obviously, we may assume w.l.o.g. that $C$ satisfies the triangle inequality and $G$ is a complete graph (define edge costs by shortest paths).

The Steiner tree problem has been investigated extensively with respect to approximation (for a recent survey, see [1]) and computational complexity, both from a theoretical and practical point of view, cf., e.g., [3] for an overview and [7]. Particular attention has been paid to the rectilinear Steiner tree problem, i.e., the case where the graph is a grid graph in the plane. For this case, which remains NP-complete [5], so-called exact algorithms have been designed [3], solving the problem in

$$
O^{*}\left(2.386^{k}\right)=O\left(2.386^{k} \text { poly }(n)\right)
$$

(Here and in the sequel we adopt the $O^{*}$-notation to indicate that polynomial factors, i.e. factors of order $O(\operatorname{pol} y(n))$ are suppressed.)

The goal of this paper is to present a modification of the well-known Dreyfus-Wagner algorithm(cf. [2] or section 2). In addition, the worst case complexity of $O^{*}\left(3^{k}\right)$ of the Dreyfus-Wagner algorithm is - as far as we know - currently still the best for solving the problem in general graphs. We shortly describe the Dreyfus-Wagner algorithm in section 2. Section 3 then presents our modification, yielding an improved worst case complexity of order $O^{*}\left(2.684^{k}\right)$. In section 4, Fößmeier and Kaufmann's algorithm is slightly modified and used as a subroutine in our algorithm to obtain a runtime of $O^{*}\left(2.335^{k}\right)$ for the rectilinear case.

\section{The Dreyfus-Wagner Algorithm}

The Dreyfus-Wagner algorithm solves the Steiner tree problem for $S \subseteq V$ by dynamic programming. More precisely, it computes optimal trees $T(X \cup v)$ for all $X \subseteq S$ and $v \in V$ recursively.

The crucial observation is as follows. Assume first that $v$ is a leaf of the (unknown) optimal tree $T(X \cup v)$. Then $v$ is joined in $T(X \cup v)$ to some node $w$ of $T(X \cup v)$ along a shortest path $P_{v w}$, such that either $w \in X$ or $w \notin X$, i.e., $w$ is a Steiner node in $T(X \cup v)$. In both cases we have $T(X \cup v)=P_{v w} \cup T(X \cup w)$. In case $w$ is a Steiner node, it splits $T(X \cup w)$, i.e., we can decompose $T(X \cup w)=T\left(X^{\prime} \cup w\right) \cup T\left(X^{\prime \prime} \cup w\right)$ for some nontrivial 
bipartition $X=X^{\prime} \cup X^{\prime \prime}$. We may thus write (abusing the notation slightly in an obvious way)

$$
T(X \cup v)=\min \quad P_{v w} \cup T\left(X^{\prime} \cup w\right) \cup T\left(X^{\prime \prime} \cup w\right),
$$

where the minimum is taken over all $w \in V$ and all nontrivial bipartitions $X=X^{\prime} \cup X^{\prime \prime}$. Note that (1) also holds in case $w \in X$ if we let $X^{\prime}=$ $X \backslash\{w\}$ and $X^{\prime \prime}=\{w\}$. Finally, note that (1) also remains valid without our assumption of $v$ being a leaf in $T(X \cup v)$. Indeed, if $v$ is an internal node of $T(X \cup v)$, we may simply take $w=v$ (and $\left.P_{v w}=\varnothing\right)$.

The recursion (1) thus allows us to compute all optimal trees $T(X \cup v)$ for $v \subseteq V$ and $X \subseteq S$ of size $|X|=i$ recursively for $i=1,2, \cdots, k$. Assuming that we have already computed all these trees up to level $i-1$, the minimum in (1) for a given $X \subseteq S$ of size $|X|=i$ can be computed in time $O^{*}\left(2^{i}\right)$. Hence, in total the algorithm takes

$$
O^{*}\left(\sum_{i=1}^{k}\left(\begin{array}{l}
k \\
i
\end{array}\right) 2^{i}\right)=O^{*}\left(3^{k}\right) .
$$

\section{Improving the Dreyfus-Wagner Algorithm}

The basic idea for improvement is as follows. We use the Dreyfus-Wagner algorithm to compute minimum Steiner tree for all subsets of $S$ of size at most $\frac{k}{2}$ (or even less), and then seek to compose the minimum Steiner tree for $S$ from these smaller trees. The basic difficulty to overcome is the following. Assume we knew that the minimum Steiner tree $T$ for $S$ contains some point $v$ whose removal splits $T$ into three branches $T^{\prime}, T^{\prime \prime}$ and $T^{\prime \prime \prime}$, connecting three corresponding subsets $S^{\prime}, S^{\prime \prime}$ and $S^{\prime \prime \prime}$ of $S$ of size approximately $\frac{k}{3}$ each. Then $v$ is the unique node splitting $T$ into components of size at most $\frac{k}{2}$. On the other hand, exhaustive search for all possible (de-) compositions of $T$ into three such subtrees amounts to search for all partitions $S=S^{\prime} \cup S^{\prime \prime} \cup S^{\prime \prime \prime}$ into sets of size $\frac{k}{3}$ (and the unknown node $v$ ). The time needed by such an exhaustive search would be

$$
\left(\begin{array}{c}
k \\
k / 3
\end{array}\right)\left(\begin{array}{c}
2 k / 3 \\
k / 3
\end{array}\right) \approx 3^{k},
$$

due to Stirling's formula. 
For this reason, the standard way of decomposing $T$ (as in the DreyfusWagner algorithm) turns out to be inadequate. We use instead the following kind of decomposition.

Definition. An $r$-split of a tree $T \subseteq E$ is a partition

$$
T=T_{1} \cup \cdots \cup T_{r}
$$

such that each

$$
T_{1} \cup \cdots \cup T_{i}, \quad i=1, \ldots, r
$$

is connected.

Now consider a fixed minimum Steiner tree $T$ for $S \subseteq V$ and an $r$-split $T=T_{1} \cup \cdots \cup T_{r}$ as above. So, $T_{1}$ is a subtree of $T$ and for $i \geq 2, T_{i} \subseteq E$ consists of several components, each of them containing exactly one node in the set $V\left(T_{i}\right) \cap\left[V\left(T_{1}\right) \cup \cdots \cup V\left(T_{i-1}\right)\right]$. More precisely, let us define

$$
\begin{array}{ll}
A_{i}^{-} & :=V\left(T_{i}\right) \cap\left[V\left(T_{1}\right) \cup \cdots \cup V\left(T_{i-1}\right)\right] \\
A_{i}^{+} & :=V\left(T_{i}\right) \cap\left[V\left(T_{i+1}\right) \cup \cdots \cup V\left(T_{r}\right)\right] \backslash A_{i}^{-} \\
A_{i}:=A_{i}^{+} \cup A_{i}^{-} & (i=1, \ldots, r) \\
S_{i}:=S \cap V\left(T_{i}\right) \backslash A_{i} &
\end{array}
$$

We refer to $A:=A_{1} \cup \cdots \cup A_{r}$ as the set of split nodes. A split node $a \in A_{i}^{-}$ connects a component of $T_{i}$ to $T_{1} \cup \cdots \cup T_{i-1}$, while $a \in A_{i}^{+}$is good for connecting a component of some $T_{j}, j>i$ to $T_{i}$. The sets $S_{i}, i=1, \ldots, n$ are pairwise disjoint and if $|A|$ is "small" compared to $k=|S|$, the $S_{i}$ are close to forming a partition of $S$. Using this kind of split-decomposition, it can be shown that $T$ has a 2 -split with $\left|S_{1}\right|,\left|S_{2}\right| \approx \frac{k}{2}$. As we will see, a (theoretically) even faster algorithm is obtained by considering certain 3-splits of $T$. Before analyzing these in detail, however, let us first state some simple facts.

Recall that we assume $G$ to be complete. For $B \subseteq V$, we denote $G / B$ the graph obtained from $G$ by identifying all vertices $b \in B$ with a new vertex $v_{B}$ (i.e., contracting all the $|B|(|B|-1) / 2$ edges induced by $B$ ). Edge costs in $G / B$ are again defined via shortest path distances.

Lemma 1 Let $T \subseteq E$ be a minimum Steiner tree for $S \subseteq V$ and let $T=T_{1} \cup \cdots \cup T_{r}$ be an $r$-split. Let $A_{i}^{ \pm}$and $S_{i}$ be defined as in (2). Then (i) $T_{1} \cup \cdots \cup T_{i}$ is a minimum Steiner tree for $S_{1} \cup \cdots \cup S_{i} \cup A_{1} \cup \cdots \cup A_{i}$.

(ii) $T_{i}$ is a minimum Steiner tree for $S_{i} \cup A_{i}^{+} \cup v_{A_{i}^{-}}$in $G / A_{i}^{-}$.

Proof. (i) Let $\tilde{T} \subseteq E$ be any tree connecting $S_{1} \cup \cdots \cup S_{i} \cup A_{1} \cup \cdots \cup A_{i}$ in $G$. Then it is straightforward from the definition of $r$-split that

$$
\tilde{T} \cup T_{i+1} \cup \cdots \cup T_{r}
$$


connects $S_{1} \cup \cdots \cup S_{r} \cup A$. But $T=T_{1} \cup \cdots \cup T_{r}$ is a minimum Steiner tree connecting $S_{1} \cup \cdots \cup S_{r} \cup A$, implying

$$
c\left(T_{1} \cup \cdots \cup T_{r}\right) \leq c\left(\tilde{T} \cup T_{i+1} \cup \cdots \cup T_{r}\right)
$$

Hence $c\left(T_{1} \cup \cdots \cup T_{i}\right) \leq c(\tilde{T})$, proving (i).

(ii) Each component of $T_{i}$ is joined to $T_{1} \cup \cdots \cup T_{i-1}$ by a (unique) common point in $A_{i}^{-}$. Therefore, $T_{i}$ is a tree in $G / A_{i}^{-}$. Furthermore, $A_{i}^{+}$is, by definition, disjoint from $A_{i}^{-}$and spanned by $T_{i}$. Summarizing, $T_{i}$ is a Steiner tree for $S_{i} \cup A_{i}^{+} \cup v_{A_{i}^{-}}$in $G / A_{i}^{-}$.

We are left to prove minimality of $T_{i}$. Let $\tilde{T}_{i} \subseteq E$ be any Steiner tree for $S_{i} \cup A_{i}^{+} \cup v_{A_{i}^{-}}$in $G / A_{i}^{-}$. Then certainly

$$
T_{1} \cup \cdots \cup T_{i-1} \cup \tilde{T}_{i} \subseteq E
$$

is connected (as $\tilde{T}_{i}$ connects to $v_{A_{i}}$ ) and spans

$$
S_{1} \cup \cdots \cup S_{i} \cup A_{1} \cup \cdots \cup A_{i-1} \cup A_{i}^{+}=S_{1} \cup \cdots \cup S_{i} \cup A_{1} \cup \cdots \cup A_{i}
$$

(as $\left.A_{i}^{-} \subseteq A_{1} \cup \cdots \cup A_{i-1}\right)$.

Hence we conclude from (i) that $c\left(T_{i}\right) \leq c\left(\tilde{T}_{i}\right)$, proving the minimality of $T_{i}$.

In what follows, we focus on 3 -splits of the minimum Steiner tree $T$ for $S \subseteq V,|S|=k$. Note that any 3 -split $T=T_{1} \cup T_{2} \cup T_{3}$ may also be considered as a 2 -split $T=T_{1} \cup\left(T_{2} \cup T_{3}\right)$. So, in particular, the following result implies the existence of a 2 -split $T=T_{1} \cup T_{2}$ with $\left|S_{1}\right| \approx\left|S_{2}\right| \approx \frac{1}{2} k$.

Theorem 2 For each $\epsilon>0$ there exists a number $M=M_{\epsilon}=O\left(\frac{1}{\epsilon}\right)$ such that the following holds: Any minimum Steiner tree $T$ for $S \subseteq V$ with $k=|S|$ large enough allows a 3-split $T=T_{1} \cup T_{2} \cup T_{3}$ with $\left|S_{1}\right|,\left|S_{2}\right| \leq(\alpha+\epsilon) k$, $\left|S_{3}\right| \leq(1-2 \alpha+2 \epsilon) k$ and $|A| \leq M$ for any prescribed value of $\alpha \leq \frac{1}{2}$.

Remark 3 The bound $M=O\left(\frac{1}{\epsilon}\right)$ can probably be improved to $M=$ $O\left(\log \frac{1}{\epsilon}\right)$. For our purposes, however, it suffices to know that for each fixed $\epsilon>0$ there is a constant upper bound on the size of $|A|$.

Proof of Theorem 2: There exists a vertex $v \in V(T)$ such that each component of $T \backslash v$ connects at most $\frac{k}{2}$ elements from $S$. Each such component connecting more than $\frac{k}{4}$ elements from $S$ can again be split in a similar way. Continuing this process, we exhibit a set $A_{0} \subseteq V(T)$ of size at most $M=\left\lceil\epsilon^{-1}\right\rceil$ such that each component $C_{j}$ of $T \backslash A_{0}$ is "small" in the sense that it connects a subset $S_{j}^{0} \subseteq S$ of at most $\epsilon k$ elements. 
We now construct $T_{1}$ by successively adding such small components, one at a time, together with the corresponding connecting nodes $a \in A_{0}$, say,

$$
V\left(T_{1}\right)=V\left(C_{1}\right) \cup \cdots \cup V\left(C_{s}\right) \cup A_{0}^{\prime}, \quad A_{0}^{\prime} \subseteq A_{0}
$$

until

$$
(\alpha-\epsilon) k \leq \sum_{j=1}^{s}\left|S_{j}^{0}\right| \leq \alpha k
$$

holds. We then construct $T_{2}$ by extending $T_{1}$ in a similar way, i.e.,

$$
V\left(T_{1} \cup T_{2}\right)=V\left(C_{1}\right) \cup \cdots \cup V\left(C_{s}\right) \cup \cdots \cup V\left(C_{t}\right) \cup A_{0}^{\prime} \cup A_{0}^{\prime \prime}
$$

with

$$
(\alpha-\epsilon) k \leq \sum_{j=s+1}^{t}\left|S_{j}^{0}\right| \leq \alpha k
$$

and set $T_{3}:=T \backslash\left(T_{1} \cup T_{2}\right)$.

Note that, by construction, $T=T_{1} \cup T_{2} \cup T_{3}$ is a 3 -split with corresponding set of split nodes $A \subseteq A_{0}$. The set $S_{1}$ consists of all $S_{j}^{0}, j=1, \cdots, s$, plus the set $S \cap A_{0}^{\prime} \backslash A$. Hence

$$
(\alpha-\epsilon) k \leq\left|S_{1}\right| \leq \alpha k+\left|A_{0}\right| \leq(\alpha+\epsilon) k
$$

for all $k \geq \frac{M}{\epsilon}=\frac{1}{\epsilon^{2}}$. Similarly,

$$
(\alpha-\epsilon) k \leq\left|S_{2}\right| \leq(\alpha+\epsilon) k
$$

holds, establishing the proof of the claim.

The algorithm. After these preliminaries, it should now be clear how to proceed. Given $\epsilon>0$ and a suitable $\alpha \leq \frac{1}{2}$ (to be determined below), we apply the Dreyfus-Wagner algorithm to compute minimum Steiner trees for all subsets of type

$$
\widetilde{S} \cup \widetilde{A}^{+} \cup v_{\widetilde{A}^{-}}, \quad \widetilde{S} \subseteq S, \quad|\widetilde{S}| \leq(\alpha+\epsilon) k
$$

in $G / \widetilde{A}^{-}$, for all disjoint subsets, $\widetilde{A}^{+}, \widetilde{A}^{-} \subseteq A$ and all $A \subseteq V$ of size at most $M$. The number of possible choices for $\widetilde{A}^{+}$and $\widetilde{A}^{-}$is bounded by $n^{M}$, which is polynomial in $n$. Assuming that $k$ is large enough, we may assume that 
$M \leq \epsilon k$, so that $|S \cup A| \leq(1+\epsilon) k$ and, similarly, $\left|\widetilde{S} \cup \widetilde{A}^{+}\right|<(\alpha+2 \epsilon) k$. So this computation takes

$$
2^{2 M} \sum_{i=2}^{(\alpha+2 \epsilon) k}\left(\begin{array}{c}
(1+\epsilon) k \\
i
\end{array}\right) 2^{i}=O^{*}\left(\begin{array}{c}
(1+\epsilon) k \\
(\alpha+2 \epsilon) k
\end{array}\right) 2^{(\alpha+2 \epsilon) k}
$$

in total.

The second part of the algorithm is an exhaustive search for the 3-split $T=T_{1} \cup T_{2} \cup T_{3}$ whose existence is assured in Theorem 3.2. Basically, this comes down to finding the associated sets $S_{i}$ (plus the corresponding sets of split nodes $A_{i}$ out of a polynomial number of possible choices). For a fixed set of split nodes $A \subseteq V$, we thus search for a partition $S \backslash A=S_{1} \cup S_{2} \cup S_{3}$ with $\left|S_{1}\right|,\left|S_{2}\right| \leq(\alpha+\epsilon) k$ and $\left|S_{3}\right| \leq(1-2 \alpha+2 \epsilon) k$. For $\alpha$ close to $\frac{1}{2}$, this takes time of order

$$
O^{*}\left(\left(\begin{array}{c}
k \\
(1-2 \alpha-2 \epsilon) k
\end{array}\right) 2^{(2 \alpha+2 \epsilon) k}\right)
$$

which also gives the total time bound for the second phase of the algorithm.

Setting $\epsilon=0$, we obtain an upper bound on the total computation time by solving

$$
\left(\begin{array}{c}
k \\
\alpha k
\end{array}\right) 2^{\alpha k}=\left(\begin{array}{c}
k \\
(1-2 \alpha) k
\end{array}\right) 2^{2 \alpha k}
$$

or, according to Stirling's Formula

$$
\left(\frac{1}{\alpha}\right)^{\alpha}\left(\frac{1}{1-\alpha}\right)^{1-\alpha}=\left(\frac{1}{2 \alpha}\right)^{2 \alpha}\left(\frac{1}{1-2 \alpha}\right)^{1-2 \alpha} 2^{\alpha} .
$$

The solution of this equation is $\alpha<0.436$. Hence we can achieve a total time bound of

$$
\left[\left(\frac{1}{0.436}\right)^{0.436}\left(\frac{1}{0.564}\right)^{0.564} 2^{0.436}\right]^{k}=2.684^{k}
$$

by an appropriately small choice of $\epsilon>0$.

\section{The rectilinear case}

Given a set $S=\left\{s_{1}, \ldots, s_{k}\right\}$ of points in the plane, the rectilinear Steiner tree problem asks for a shortest tree connecting the points in $S$, relative to the so-called Manhattan-metric (where the distance between two points is, by 
definition, the sum of the differences of their $x$ - and $y$-coordinates). Equivalently, we may define an instance of the Steiner tree problem rectilinear, if the underlying graph $G=(V, E)$ is a grid graph (the so-called "Hannan-grid") in the plane (with the grid being generated by the $x$-resp. $y$-coordinates of the points in $S$ ). We refer the reader to [8] for an introduction to the rectilinear case.

According to Ganley and Cohoon [4] and Fößmeier and Kaufmann [3], the dynamic programming approach for computing minimum Steiner trees can be implemented more efficiently in the rectilinear case as follows. The basic notion is that of a full Steiner tree: If $X \subseteq S$ is given, a minimum Steiner tree $T=T(X)$ for $X$ is full if each node in $X$ is a leaf of $T$. We call $X \subseteq S$ a full set if every minimum Steiner tree for $X$ is full.

Clearly, every minimum Steiner tree $T=T(X)$ for $X \subseteq S$ decomposes uniquely into full components, i.e., edge-disjoint full subtrees. A crucial result of Hwang [6] states that, in the rectilinear case, full components (sets) can be assumed to have a certain simple topological structure. Subsets $X \subseteq S$ with this particular structure are called candidate full sets. The set of all candidate full sets $X \subseteq S$ is denoted by $\mathcal{F}(S)$. Given a candidate full set $X \in \mathcal{F}(S)$, one can (due to the particular simple structure of full components) easily compute (in linear time) a corresponding candidate full tree $T_{\text {full }}(X)$, which is guaranteed to be a minimum Steiner tree for $\mathrm{X}$ in case $X$ is a full set.

Adopting the notation

$$
X=X_{1} \bowtie X_{2} \Leftrightarrow X=X_{1} \cup X_{2},\left|X_{1} \cap X_{2}\right|=1
$$

from [4], we may thus compute minimum Steiner trees for all $X \subseteq S$ by means of the recursion

$$
T(X)=\min T_{\text {full }}\left(X_{1}\right) \cup T\left(X_{2}\right),
$$

where the minimum is taken over all decompositions $X=X_{1} \bowtie X_{2}$ with $X_{1} \in \mathcal{F}(S)$ and $\left|X_{1}\right| \geq 2$. Note that when $X \subseteq S$ itself is a full set, then $X \in \mathcal{F}(S)$, so we may take $X_{1}=X$ and let $X_{2}$ be a singleton.

The running time of this procedure depends on the number of candidate full sets. Indeed, letting

$$
\mathcal{F}(X):=\left\{X_{1} \in \mathcal{F}(S) \mid X_{1} \subseteq X\right\}
$$

we find that computing the minimum in (3) takes time $O^{*}(|\mathcal{F}(X)|)$ - assuming recursively that $T\left(X_{2}\right)$ is known already for all subsets $X_{2} \in S$ of size 
$\left|X_{2}\right|<|X|$. (Recall that, as mentioned above, $T_{\text {full }}\left(X_{1}\right)$ can be computed for given $X_{1} \in \mathcal{F}(S)$ in time $O\left(\left|X_{1}\right|\right)=O(k)=O^{*}(1)$.)

The main result of Ganley and Cohoon [4] states that (due to the specific topological structure of full sets), only very few subsets of $X$ are candidate full sets. More precisely, they show that for $|X|=i$ we have $|\mathcal{F}(X)| \leq 1.62^{i}$. This bound is further improved by Fößmeier and Kaufmann [3] to $|\mathcal{F}(X)| \leq$ $1.386^{i}$. As a consequence, the total running time of the recursion, applying (3) to all sets $X \subseteq S$ with increasing size $|X|=i$, can be bounded by

$$
O^{*}\left(\sum_{i=1}^{k}\left(\begin{array}{l}
k \\
i
\end{array}\right) 1.386^{i}\right)=O^{*}\left(2.386^{k}\right) .
$$

Applying our splitting technique to this recursion, we would-just like in section 3-compute the minimum Steiner trees only up to a certain level $i=\alpha k, \alpha<\frac{1}{2}$. The time consumed by this computation is

$$
O^{*}\left(\sum_{i=1}^{\alpha k}\left(\begin{array}{c}
k \\
i
\end{array}\right) 1.386^{i}\right)=O^{*}\left(\left(\begin{array}{c}
k \\
\alpha k
\end{array}\right) 1.386^{\alpha k}\right) .
$$

On the other hand, searching for the unknown 3-split would roughly (we set $\epsilon=0)$ take

$$
O^{*}\left(\left(\begin{array}{c}
k \\
(1-2 \alpha) k
\end{array}\right) 2^{2 \alpha k}\right)
$$

Again, the best upper bound on the running time of our algorithm is obtained by balancing (5) and (6). For $\alpha \approx 0.477$, we obtain an upper bound of $O^{*}\left(2.335^{k}\right)$ - a minor improvement over the original bound (4).

There is one problem that we are left to solve: Recall that in "phase 1 " of our algorithm we compute small Steiner trees up to level $i=\alpha k$ not only in $G$, but also in certain contracted graphs. But these graphs are in general not rectilinear anymore! A moment's thought, however, reveals that this problem as simply non-existent. Indeed, the only reason for considering contracted graphs in section 3 is notational convenience: Assume, for example, that we are to compute (recursively) the minimum Steiner tree for a certain subset

$$
X \cup v_{A} \quad \text { in } G / A, \quad X \subseteq V \backslash A .
$$

This is tantamount to looking for a minimum Steiner A-forest for $X$ in $G$, i.e., a minimum length forest $F \subseteq E$, connecting all of $X$ to $A$. In other 
words, a Steiner $A$-forest for $X$ consists of $|A|$ tree components $(|A| \geq 1)$, each containing exactly one node of $A$. Thus a minimum Steiner $A$-forest $\mathcal{F} \subseteq E$ gives rise to a minimum Steiner tree $F$ in $G / A$ and conversely.

Rather than computing minimum Steiner trees for various sets $X \cup v_{A}$ in certain contracted graphs $G / A$, we compute minimum Steiner $A$-forests in $G$ for various sets $X$ and $A$. In the rectilinear case, this can be done in complete analogy to the full set dynamic programming approach described above.

For $X \subseteq S \backslash A,|A| \geq 1$, let $F_{A}(X \cup A)$ denote the minimum Steiner $A$-forest for $X$. Then $F_{A}(X \cup A)$ consists of at most $|A|$ nonempty tree components. (Recall that we always consider a tree as a set of edges. So a tree component consisting of a single vertex $a \in A$ is empty.) Each such nonempty tree component contains exactly one node $a \in A$ and decomposes into one or more full components. Thus we can compute $F_{A}(X \cup A)$ recursively from $F_{A}(A)=\varnothing$ and

$$
F_{A}(X \cup A)=\min T_{\text {full }}\left(X_{1}\right) \cup F_{A}\left(X_{2} \cup A\right)
$$

where the minimum is taken over all candidate full sets $X_{1} \in \mathcal{F}(X \cup A)$ with $X \cup A=X_{1} \bowtie\left(X_{2} \cup A\right)$.

Note that the dynamic program (7) is (for fixed $A$ ) very similar to (3). (Indeed, we formally obtain (4) from (7) by setting $A=\varnothing$.) This completes our proof of the upper bound on the running time.

\section{Concluding remarks}

We presented a splitting technique to speed up the dynamic programming approach to minimum Steiner tree computation. We do not claim that our improvements as presented in sections 3 and 4 are of any practical use. Yet it might turn out that already for small values of $|A|$, say $|A|<4$, the existence of 2-splits with $\left|S_{i}\right|$ fairly close to $k / 2$ can be guaranteed. This needs to be further investigated, as it might well be of practical interest.

\section{References}

[1] C. Gröpl, S. Hougardy, T. Nierhoff and H. J. Prömel, (2001) Approximation Algorithms for the Steiner Tree Problem in Graphs, in: Steiner Trees in Industries, X. Cheng and D.-Z. Du (eds.), Kluwer. 
[2] S. E. Dreyfus and R. A. Wagner, (1972) The Steiner problem in graphs. Networks, 1, 195-207.

[3] U. Fößmeier and M. Kaufmann, (2000) On exact solutions for the rectilinear Steiner tree problem Part 1: Theoretical results. Algorithmica, 26, 68-99.

[4] J. L. Ganley and J. P. Cohoon, (1994) Optimal rectilinear Steiner minimal trees in $O\left(n^{2} 2.62^{n}\right)$ time, in: Proceedings of the Sixth Canadian Conference on Computational Geometry, 308-313.

[5] M. Garey and D. Johnson, (1977) The Rectilinear Steiner Tree Problem is NP-Complete, SIAM Journal on Applied Mathematics 32(4), 826-834.

[6] F. K. Hwang, (1976) On Steiner Minimal Trees with Rectilinear Distance. SIAM Journal on Applied Mathematics, 30(1), 104-114.

[7] D. M. Warme, P. Winter and M. Zachariasen, (2000) Exact Algorithms for Plane Steiner Tree Problems: A Computational Study, in: Advances in Steiner Trees, D.Z. Du, J.M. Smith and J.H. Rubinstein (eds.), Kluwer.

[8] M. Zachariasen, The Rectilinear Steiner Tree Problem: A Tutorial, in: Steiner Trees in Industries, X. Cheng and D.-Z. Du (eds.), Kluwer. 